\title{
25 Research Square \\ Brief Report: Ionising Radiation Induces Autophagy in the Adult Drosophila Brain and Intestine
}

\section{Terrence M Trinca}

Cardiff University

Bilal R Malik ( $\nabla$ bilalrashidmalik@gmail.com )

Cardiff University https://orcid.org/0000-0001-5522-4990

\section{Research Article}

Keywords: Ionising Radiations, autophagy, Drosophila, cancer, gamma radiation

Posted Date: February 8th, 2022

DOI: https://doi.org/10.21203/rs.3.rs-1283711/v1

License: (9) This work is licensed under a Creative Commons Attribution 4.0 International License.

Read Full License 


\section{Abstract}

Exposure to various genotoxic and oxidative stresses can induce changes in the internal state of a cell leading to cell death. Ionizing radiation is routinely used in clinics to treat various types of cancers, with secondary effects sometimes damaging internal organs particularly the gut. Drosophila melanogaster has become one of the most trusted model organisms to study human disease and various biological pathways. Here we have used gamma radiation to determine for the first time whether they have an effect on autophagy in two different tissues in Drosophila; the gut and the brain. We find that in both tissues exposure to radiation leads to an increase in autophagy.

\section{Introduction}

Maintaining a healthy cellular homeostasis is crucial for the survival of animals. This requires the ability to protect cells and cellular components from damage from toxins and various sources of mutagens including chemicals and radiation since we cannot avoid them completely as we sometimes use them to our advantage, e.g. we use chemicals to treat diseases and radiations to treat cancers. The effect of these mutagens and toxins vary according to their targets, all leading to some level of cellular stress. While the genotoxic effects of radiation are well known, their effects on other vital cellular processes such as autophagy are much less known.

Radiation has been used in medicine for over a century with varied application [1, 2]. In modern medicine, radiation is used mostly for the treatment of cancers, and it is estimated it should be administered to approximately $52 \%$ of all cancer patients [3]. Yet the effects of radiation on the surrounding tissues is unavoidable and can lead to a range of short- and long-term side effects including hematopoietic and gastrointestinal syndrome, cardiovascular and nervous system syndromes and a range of other bystander effects [4-7]. Once a cell has been exposed to high doses of radiation, which are required for treatment of cancers, they respond by activation of a signalling cascade either leading to cell cycle arrest or apoptosis.

Radiation-induced free radical generation can lead to oxidative stress in the cell which predisposes it to apoptosis. Unless the stress molecules are quenched (such as ROS quenching by antioxidants), they may lead to protein oxidation and subcellular organelle damage. If unchecked, these damages can lead the cell to undergo apoptosis. Other responses to radiation exposure may include inflammation of the surrounding tissues, signalling macrophages and other immune responsive cells to the site of injury further escalating the damage response $[8,9]$.

Once a cell has incurred some organelle or protein damage, it may respond by activation of autophagy, degrading and removing the damaged particles. This however requires functional lysosomes that contain the hydrolases on which autophagy depends. The damaged substrates are recognised by the cell and tagged for degradation. Failure to do so can result in the cell building up damaged materials such as oxidised proteins and lipids which can then interfere with the normal functioning of the cell [10]. 
Radiation cause hydrolysis of water, producing ROS, which interact with macromolecules and induce oxidative damage and stress [11]. Radiation-induced oxidative damage has been extensively studied in humans, and ROS imbalances have been shown to persist after treatment [12]. From looking at the Drosophila research, it is clear that radiation induces oxidative stress, at least short-term. Genes such GstT4 and GstD1 involved in oxidative metabolism have been shown to have increased expression post irradiation $[13,14]$. ROS imbalance is capable of inducing autophagy through the mTOR-AMPK pathway [15].

While it is well known that exposure to radiation leads to activation of the apoptotic pathway [16], nothing is known about how radiations affect autophagy, one of the cells most common response to toxins and damage $[17,18]$. Here we used Drosophila melanogaster as a model system to evaluate the effect of radiation exposure on autophagy. We find that radiation exposure leads to an increase in the levels of autophagy in two distinct cell types in Drosophila indicating that the initial response to radiation might be modulated through autophagy.

\section{Materials And Methods}

\section{Drosophila Stocks and Husbandry}

Drosophila were raised at $25^{\circ} \mathrm{C}$ on standard cornmeal/molasses/agar media. The following Drosophila stocks were used: uas-mCherry::Atg8 (gift from T. Neufeld), GMR61G12-Gal4 (Flylight collection, Jenelia Farm), Myo1A-Gal4 (gift from J. de Navascués) and uas-mCD8::GFP(Bloomington Stock Centre).

\section{lonizing radiation exposure}

3 to 6-day old flies were collected in vials containing cornmeal medium. A group of these was exposed to gamma irradiation using a Cesium-137 y-ray irradiator for a total of $150 \mathrm{~Gy}$, administered at $0.43 \mathrm{~Gy} / \mathrm{min}$. Control group was kept in the same room for the duration of gamma ray exposure.

\section{Dissections, Imaging and analysis}

Flies expressing UAS-mCherry::Atg8 and UAS-mCD8::GFP driven by GMR61G12Gal4 were dissected as described previously [19]. Briefly, fly brains were dissected from 3 to 6-day old flies in cold PBS and fixed in $4 \%$ paraformaldehyde for $30 \mathrm{~min}$. The tissues were then washed three times with PBST (10 min each), mounted in vectashield and stored at $4^{\circ} \mathrm{C}$ until imaged. Images were captured using Zeiss Spinning Disc Confocal (Cell Observer) microscope and a 63X oil immersion objective (numerical aperture 1.3).

Midguts were dissected from 3 to 6-day old flies directly into ice-cold PBS, fixed in a formalin (4\%) solution under a heptane phase for $15 \mathrm{~min}$. Tissue was permeabilised using methanol (100\%) for $15 \mathrm{~min}$. Permeabilised tissue was then blocked $3 x$ for 15 min each using PBS containing $0.1 \%$ Triton-X 100 (PBT) and $2 \%$ bovine serum albumin (BSA). Tissue was stained with the primary antibody Rabbit anti-RFP (Takara 632496) overnight ( $16 \mathrm{hrs}$ ) at $4^{\circ} \mathrm{C}$ with mild rocking, followed by washing in PBT ( $3 x$ rinses and $3 x$ washes). Tissue was stained with the secondary antibody Donkey anti-Rabbit-A594 (Thermo Scientific 
A21207) for $2 \mathrm{hr}$ at room temperature with mild rocking. DNA was stained with Hoescht at 1:5,000 (Sigma Aldrich B2261, stock solution at $10 \mathrm{mg} / \mathrm{ml}$ ) which was added alongside secondary antibodies. Tissue was washed with PBT and mounted in home-made mounting medium (Glycerol:PBS 80:20 with $4 \%$ propyl gallate). Confocal stacks were obtained in a Zeiss LSM 710 with an EC Plan16 Neofluar 40X and $63 \mathrm{X}$ oil immersion objectives (numerical aperture 1.3). All stack positions were acquired in the posterior midgut with same laser intensities and exposure time. Images were processed using FIJI [20] and statistical analysis was performed using Graphpad (PRISM 9.0).

\section{Results}

\section{Effect of Radiation on gut}

To assess the effect of gamma irradiation on the gut, we used UAS-mCherry Atg8 as a marker. We exposed a group of flies expressing mCherry-tagged Atg8 in the gut (Myo1A-Ga/4>UAS-mCherry::Atg8). Irradiation did not cause any lethality nor any other gross defects in flies until they were dissected. In comparison to controls, the gamma irradiated flies showed an increase in Atg8 levels in the gut one day after irradiation (t-test, $p<0.008$ ) (Figure 1).

\section{Effect of radiation on the brain}

To determine the effect of irradiation on adult fly brain we generated flies expressing mCherry-tagged Atg8 in a small subset of neurons in the central brain which normally express in the Pigment dispersing factor ( $p d f$ ) using GMR61G12-Ga/4. These neurons are much bigger than the rest of brain neurons and could be easily imaged. This way we were able to focus on a small subset of neurons to look for changes in the mCherry signal within individual neurons. In comparison to the controls, we observed a significant increase in the Atg8 levels indicating an increase in autophagy levels (Figure 2).

\section{Discussion}

Gamma irradiation is used as a curative measure for treatment of various cancers. The effect of this ionising radiation on different tissues and their radiosensitivity is an important factor which could help determine the optimum dosage for a given patient. The intracellular damage caused by repetitive exposure to such radiation poses a threat to patient's health who are already weakened by the impact of the disease. Knowing the full scale of impact of radiation on cellular mechanisms can help design strategies which can be used to minimise the negative impact of radiations.

Cellular response to oxidative stress includes a waste-clearance mechanism, known as autophagy, which depends on a cascade of events leading to isolation of the damaged subcellular substrates and their degradation by the lysosomal enzymes. The effect of gamma radiations on this mechanism, so crucial for survival from cellular damage, is yet unknown. We show that the cellular autophagy is significantly increased in response to gamma irradiation in two distinct types of cells in young Drosophila. While the cellular morphology and the gut intactness in the time scale of our experiment is unaffected, the increase 
in autophagy might indicate an early response to the gamma radiation. The consequence of increased autophagy can be a determining factor for cellular survival. Significantly high damage to the cellular cytoplasm and DNA could lead to overpowering of the autophagic mechanisms leading to cell death. Since the central nervous system neurons have several supporting tissues, such as microglia and oligodendrocytes, which help clear some of the secreted waste materials produces by stressed neurons, it is pertinent to determine the impact of gamma radiation on these cell types. In addition, to better understand the consequence of increased autophagy and whether the increased levels of autophagy actually represent a blockage of autophagic flux or a stimulation of autophagy, further investigation is required.

\section{Declarations}

\section{Competing Interests}

The authors have no competing interests to disclose.

\section{Funding}

This work was supported by ISSF Wellcome fellowship to Bilal Malik and a KESS2 Welsh government/Tenovus studentship to Terrence Trinca.

\section{Author Contributions}

BM conceptualised and designed the experiments. BM and TT prepared materials,, collected and analysed data and wrote the manuscript. Both authors read and approved the final manuscript.

\section{Data Availability}

The data associated with this manuscript is available from the authors on request.

\section{Ethics approval}

No ethical approvals were needed for the work.

\section{Consent to participate and publish}

No human subjects were involved in this work and hence there was no need for consent. 


\section{Acknowledgements:}

We would like to thank members of the fly groups at Cardiff for their discussions and input.

\section{References}

1. Donya M, Radford M, ElGuindy A, Firmin D, Yacoub MH (2014) "Radiation in medicine: Origins, risks and aspirations," Glob Cardiol Sci Pract, vol. no. 4, pp. 437-448, 2014, doi: 10.5339/gcsp.2014.57

2. Pusey W, Allen (1900) Roentgen rays in the treatment of skin diseases and for the removal of hair". J Cutan Dis Including Syphilis 18:302-2018

3. Delaney G, Jacob S, Featherstone C, Barton M (2005) "The role of radiotherapy in cancer treatment: Estimating optimal utilization from a review of evidence-based clinical guidelines," Cancer, vol. 104, no. 6, pp. 1129-1137, Sep. doi: 10.1002/cncr.21324

4. Gómez-Millán J et al (Mar. 2012) The importance of bystander effects in radiation therapy in melanoma skin-cancer cells and umbilical-cord stromal stem cells. Radiother Oncol 102(3):450-458. doi: 10.1016/j.radonc.2011.11.002

5. Joye I, Haustermans K (2014) “Early and Late Toxicity of Radiotherapy for Rectal Cancer. In: Otto F, Lutz MP (eds) " in Early Gastrointestinal Cancers II: Rectal Cancer, vol 203. Springer International Publishing, Cham, pp 189-201. doi: 10.1007/978-3-319-08060-4_13.

6. Ohri N, Dicker AP, Showalter TN (2012) "Late toxicity rates following definitive radiotherapy for prostate cancer," Can J Urol, vol. 19, no. 4, pp. 6373-6380, Aug.

7. Zelefsky MJ, Chan H, Hunt M, Yamada Y, Shippy AM, Amols H (2006) "Long-Term Outcome of High Dose Intensity Modulated Radiation Therapy for Patients With Clinically Localized Prostate Cancer," Journal of Urology, vol. 176, no. 4, pp. 1415-1419, Oct. doi: 10.1016/j.juro.2006.06.002

8. McKelvey KJ, Hudson AL, Back M, Eade T, Diakos $\mathrm{Cl}$ (2018) "Radiation, inflammation and the immune response in cancer," Mamm Genome, vol. 29, no. 11-12, pp. 843-865, Dec. doi: 10.1007/s00335-018-9777-0

9. Yahyapour R et al (Dec. 2018) Radiation-induced inflammation and autoimmune diseases. Military Med Res 5(1). doi: 10.1186/s40779-018-0156-7

10. Xie $L$ et al (Aug. 2019) Modes of action and adverse effects of gamma radiation in an aquatic macrophyte Lemna minor. Sci Total Environ 680:23-34. doi: 10.1016/j.scitotenv.2019.05.016

11. Azzam El, Jay-Gerin J-P, Pain D (Dec. 2012) lonizing radiation-induced metabolic oxidative stress and prolonged cell injury. Cancer Lett 327:1-2. doi: 10.1016/j.canlet.2011.12.012

12. Robbins MEC, Zhao W (2004) "Chronic oxidative stress and radiation-induced late normal tissue injury: a review," Int J Radiat Biol, vol. 80, no. 4, pp. 251-259, Apr. doi:

10.1080/09553000410001692726

13. Kuzin BA et al (2014) Combination of hypomorphic mutations of the Drosophila homologues of aryl hydrocarbon receptor and nucleosome assembly protein family genes disrupts morphogenesis, 
memory and detoxification. PLoS ONE 9(4):e94975. doi: 10.1371/journal.pone.0094975

14. Moskalev A et al (Dec. 2015) A comparison of the transcriptome of Drosophila melanogaster in response to entomopathogenic fungus, ionizing radiation, starvation and cold shock. BMC Genomics 16:S. doi: 10.1186/1471-2164-16-S13-S8

15. He L, He T, Farrar S, Ji L, Liu T, Ma X (2017) Antioxidants Maintain Cellular Redox Homeostasis by Elimination of Reactive Oxygen Species. Cell Physiol Biochem 44(2):532-553. doi:

$10.1159 / 000485089$

16. Sudmeier LJ, Howard SP, Ganetzky B (2015) "A Drosophila model to investigate the neurotoxic side effects of radiation exposure," Dis Model Mech, vol. 8, no. 7, pp. 669-677, Jul. doi: $10.1242 / \mathrm{dmm} .019786$

17. Kemp MG, Immunity I (2017) " J Cell Death, vol. 10, p. 117967071668508 , Jan. doi: $10.1177 / 1179670716685085$

18. Li F, Lang F, Zhang H, Xu L, Wang Y, Hao E (2016) "Role of TFEB Mediated Autophagy, Oxidative Stress, Inflammation, and Cell Death in Endotoxin Induced Myocardial Toxicity of Young and Aged Mice," Oxidative Medicine and Cellular Longevity, vol. pp. 1-10, 2016, doi: 10.1155/2016/5380319

19. Malik BR, Gillespie JM, Hodge JJL (2013) CASK and CaMKII function in the mushroom body a'/ $\beta^{\prime}$ neurons during Drosophila memory formation. Front Neural Circuits 7. doi: 10.3389/fncir.2013.00052

20. Schindelin J et al (Jul. 2012) Fiji: an open-source platform for biological-image analysis. Nat Methods 9(7):676-682. doi: 10.1038/nmeth.2019

\section{Figures}


Figure 1.

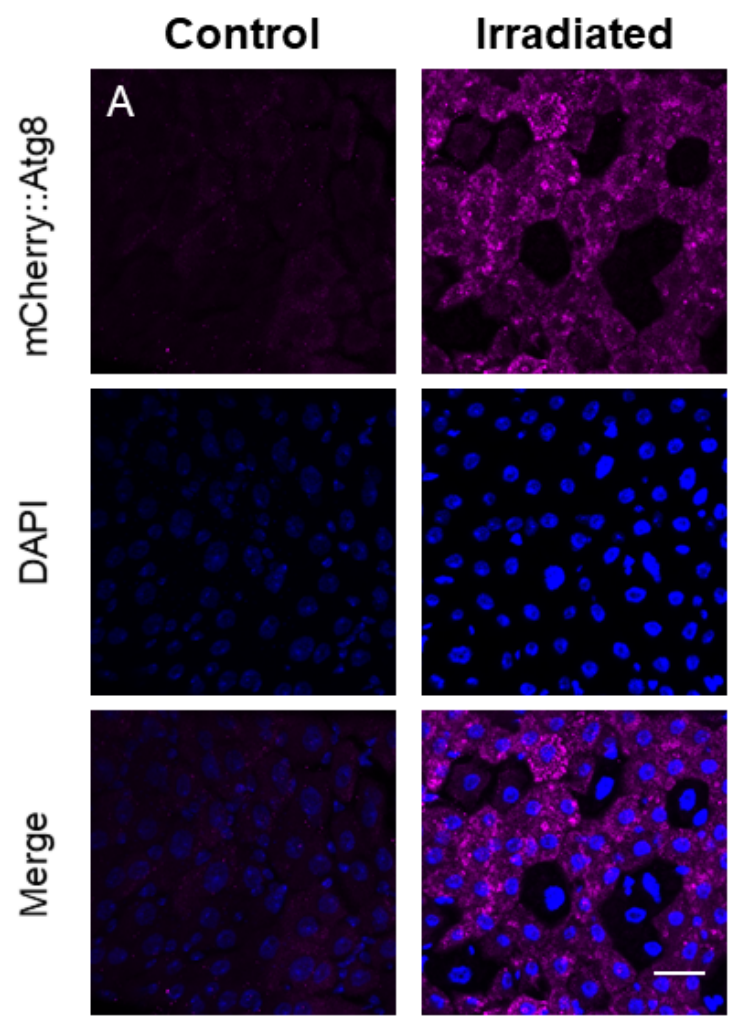

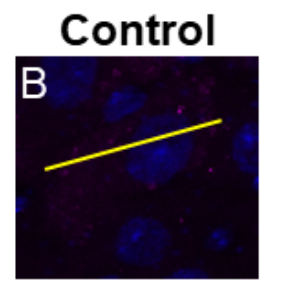

Irradiated
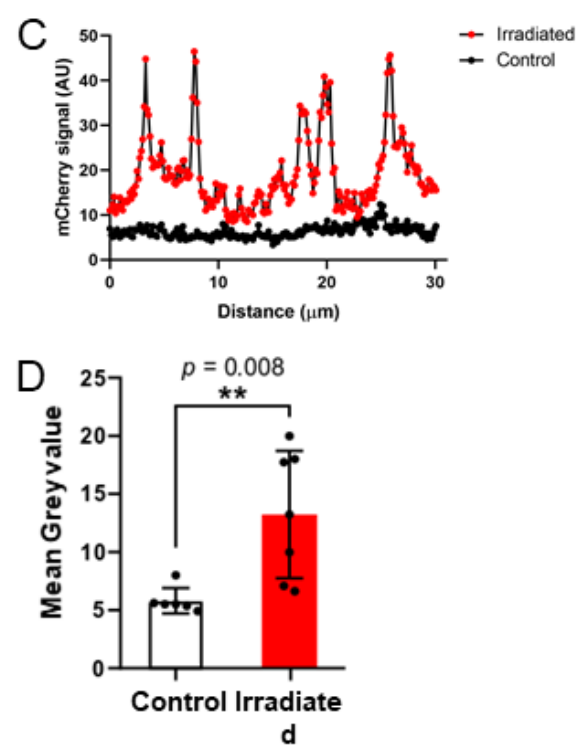

\section{Figure 1}

Effects of irradiation on gut autophagy. (A) Representative maximum projections of irradiated and control Drosophila gut . Scale bar is 20 microns. (B) Digitally magnified close-ups of cells, yellow line represents sampling path for panel (C). (C) mCherry intensity value per pixel along paths that were drawn in panel. (D) Mean grey value of mCherry in control and experimental tissue. (t-test, $n>8)(D)$. 


\section{Figure 2}

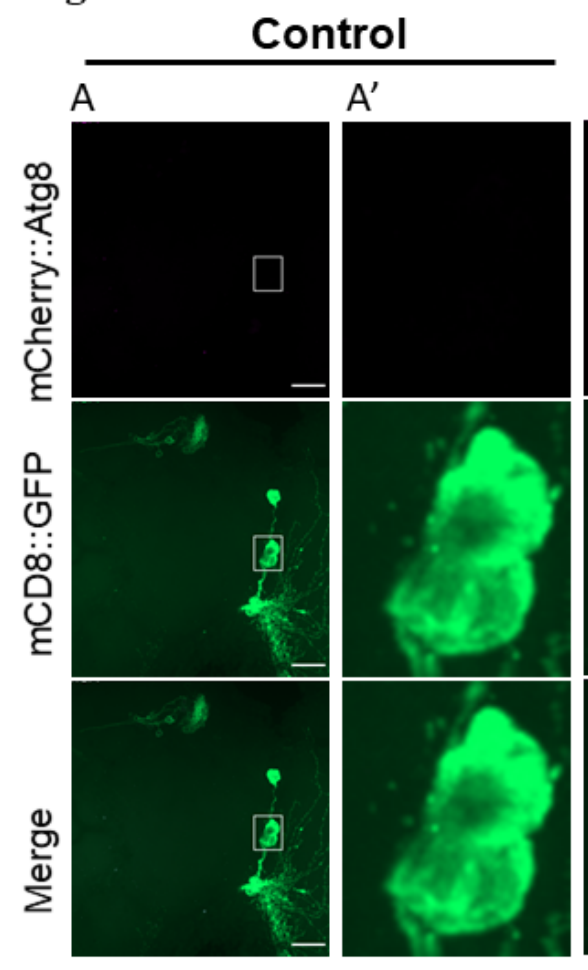

Experiment-124

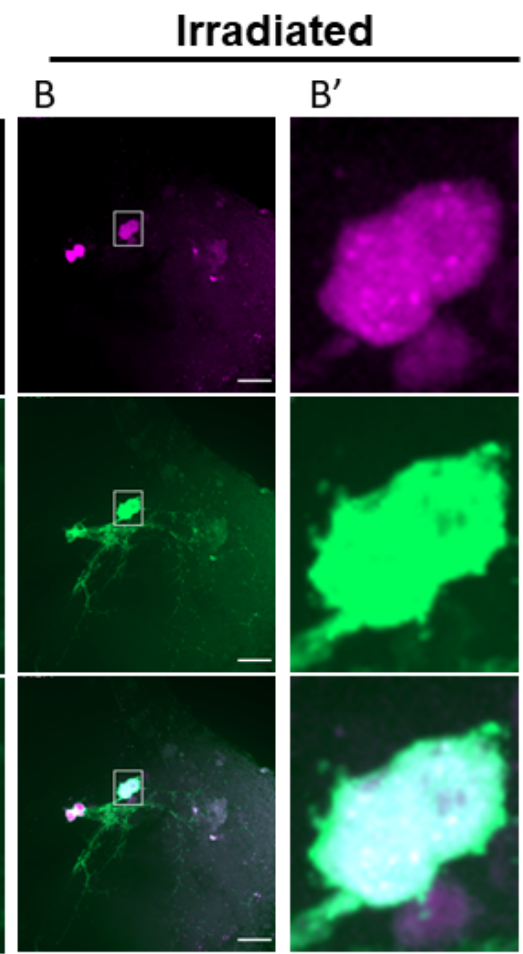

Experiment-131-2

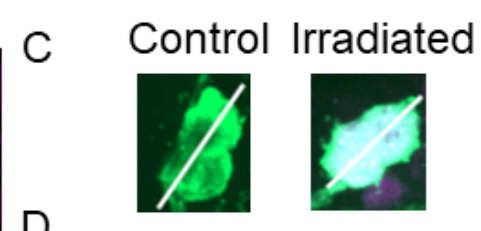

D

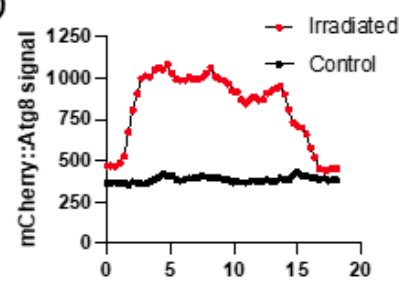

$\mathrm{E}$

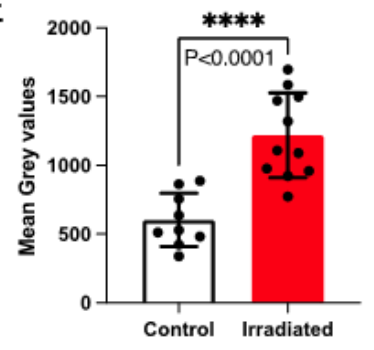

\section{Figure 2}

Autophagy is increased in response to Gamma irradiation. In comparison to control (A) brains the brains from irradiated flies (B) show increases levels of autophagy marker mCherry::Atg8 in pdf-expressing neurons in the brain. (B). Projections of representative images of the adult brain highlighting the neurons considered for measurement. Scale bar is 20 microns. ( $A^{\prime}$ and $B^{\prime}$ ) Digitally magnified close-ups of neurons. (C) Representative images of neurons, solid lines indicate sampling path for mCherry signal sampling presented in panel D. (D) mCherry signal profiles along a selected path. (E) Mean grey value of $m$ Cherry signal in control and irradiated fly neurons (t-test, $n \geq 8$ ). 